\title{
SGLT2 Inhibitors: Emerging Roles in the Protection Against Cardiovascular and Kidney Disease Among Diabetic Patients
}

This article was published in the following Dove Press journal: International Journal of Nephrology and Renovascular Disease

\author{
George Vasquez-Rios (D) \\ Girish N Nadkarni ${ }^{1,2}$ \\ 'Division of Nephrology, Department of \\ Medicine, Icahn School of Medicine at \\ Mount Sinai, New York, New York, USA; \\ ${ }^{2}$ The Charles Bronfman Institute of \\ Personalized Medicine, Icahn School of \\ Medicine at Mount Sinai, New York, \\ New York, USA
}

Purpose of Review: Type 2 diabetes mellitus (T2DM) is a prevalent disease with the severe clinical implications including myocardial infarction, stroke, and kidney disease. Therapies focusing on glycemic control in T2DM such as biguanides, sulfonylureas, thiazolidinediones, and insulin-based regimens have largely failed to substantially improve cardiovascular and kidney outcomes. We review the recent findings on sodium-glucose cotransporter type 2 (SGLT2) inhibitors which have shown to have beneficial cardiovascular and kidney-related effects.

Recent Findings: SGLT2 inhibitors are a new class of diabetic medications that reduce the absorption of glucose in the kidney, decrease proteinuria, control blood pressure, and are associated with weight loss. SGLT2 inhibitors provide complementary therapy independent of insulin secretion or action with proved glucose-lowering effects. Recent placebo-controlled clinical trials have demonstrated that these medications can decrease cardiovascular death, progression of kidney disease, and all-cause mortality in diabetic and non-diabetic patients. Interestingly, SGT2 inhibitors such as dapagliflozin have also proven to decrease heart failure admissions and cardiovascular endpoints in non-diabetic patients, suggesting pleiotropic effects. The exact mechanisms responsible for reductions in atherosclerotic heart disease, need for kidney replacement therapy, and progressive kidney disease remain unknown. While regulation of glomerular hyperfiltration, albuminuria, and natriuresis may be part of the explanation, it is possible that complex cellular effects including energy balance optimization, downregulation of oxidative stress, and modulation of pro-inflammatory signaling pathways are associated with favorable outcomes observed in large clinical studies.

Conclusion: SGLT2 inhibitors are novel antidiabetic medications with immense utility in the management of patients with T2DM. Furthermore, SGLT2 inhibitors have demonstrated to reduce the progression to advanced forms of kidney disease and its associated complications. These medications should be front and center in the management of patients with diabetic kidney disease with and without chronic kidney disease as they confer protection against cardiovascular/renal death and improve all-cause mortality. Future studies should evaluate the benefits and implications of early initiation of SGLT2 inhibitors, as well as the long-term effects of this therapy.

Keywords: hyperglycemia, SGLT2, renal failure, heart disease, coronary artery disease

\section{Introduction}

Type 2 Diabetes Mellitus (T2DM) is a highly prevalent condition that confers significant morbidity and mortality among cardiovascular and renal patients. Results from the International Diabetes Federation Diabetes Atlas (9th edition) reveal that the 2019
Icahn School of Medicine at Mount Sin

One Gustave L Levy Place, Box 1243

New York, NY 10029, USA

Tel $+|-2| 2-24|-| 385$

Fax +I-2I2-849-2643

Email girish.nadkarni@mountsinai.org
International Journal of Nephrology and Renovascular Disease 2020:13 28I-296 
Table I Potential Mechanisms of Action of SGLT2 Inhibitors in the Cardio-Renal Physiology

\begin{tabular}{|c|c|}
\hline Kidney & Heart \\
\hline $\begin{array}{l}\text { Regulation of cellular stress mediated by energy unbalance (reduction } \\
\text { of ATP-dependent sodium/glucose uptake) }\end{array}$ & $\begin{array}{l}\text { Regulation of cellular stress mediated by energy unbalance. Adaptation to } \\
\text { alternative energy fuels (e.g. fatty acids) }\end{array}$ \\
\hline $\begin{array}{l}\text { Reduction of reactive oxygen species and markers of cellular injury in } \\
\text { diabetic kidney disease. }\end{array}$ & $\begin{array}{c}\text { Reduction of reactive oxygen species and markers of cellular injury } \\
\text { during myocardial infarction and heart failure. }\end{array}$ \\
\hline $\begin{array}{l}\text { Downregulation of PKC, mTOR and TGF- } \beta \text { related-pathways. } \\
\text { Reductions in fibrosis and inflammatory cytokines. }\end{array}$ & $\begin{array}{l}\text { Modulation of JAK-STAT signaling pathways during ischemia-reperfusion } \\
\text { injury, AMPK amplification, NHEI inhibition. }\end{array}$ \\
\hline $\begin{array}{l}\text { Reduction in albuminuria, natriuresis, modulation of TGF and } \\
\text { decrements in the rate of GFR decline }\end{array}$ & Blood pressure and weight reductions \\
\hline $\begin{array}{l}\text { Decrease cellular hypoxia, increments of hematocrit and restoration of } \\
\text { EPO producing macrophages }\end{array}$ & $\begin{array}{l}\text { Diuretic effect, and volume reduction. Greater benefit when associated } \\
\qquad \text { with ACEi/ARBs }\end{array}$ \\
\hline
\end{tabular}

Abbreviations: ATP, adenosine-triphosphate; PKC, protein-kinase C; mTOR, mammalian target of rapamycin; TGF- $\beta$, transforming growing factor $\beta$; JAK-STAT, Janus kinase (JAK)-signal transducer and activator of transcription; AMPK, adenosine monophosphate activated protein kinase; NHEI, sodium-hydrogen exchanger I; TGF, tubuloglomerular feedback; GFR, glomerular filtration rate; EPO, erythropoietin; ACEi, angiotensin converting enzyme inhibitor; ARB, angiotensin receptor blockers.

estimated global prevalence of diabetes mellitus was $9.3 \%$, translated in 463 million people affected by this condition. ${ }^{1}$ Furthermore, there is a projected rise of this disease to 578 million by 2030. T2DM is the leading cause of chronic kidney disease (CKD) and end-stage kidney disease (ESKD) worldwide ${ }^{2}$ and diabetic kidney disease (DKD) has been related to the death of $>400000$ patients in both industrialized and developing countries. ${ }^{3}$

Relative insulin deficiency, insulin resistance and abnormal glucose metabolism are all factors implicated in the pathophysiology of T2DM. ${ }^{4}$ Chronic hyperglycemia induces pathological microvascular and macrovascular changes in the form of myocardial infarction (MI), stroke, and DKD. However, an increasing body of evidence suggests that complex cellular processes including endothelial damage, immune system dysregulation, and marked production of reactive oxygen species (ROS) play an important role in pathogenesis and deleterious effects of T2DM. Despite this, societal guidelines have focused on controlling hyperglycemia to prevent disease progression and end-organ complications by recommending periodic monitoring of glycated hemoglobin A1c (A1c). Thus, asides from lifestyle modifications, metformin (biguanide) is recommended as the mainstay of therapy followed sulfonylureas and thiazolidinediones. Adjuvant insulin therapy is also indicated for severe cases of persistent hyperglycemia to achieve an Alc goal $<7 \%$. However, strategies aiming to manage hyperglycemia by increasing glucose uptake (insulin, biguanides, and thiazolidinediones) or to increase the net insulin production (sulfonylureas) have been found to provide marginal benefits. ${ }^{5-7}$ Furthermore, these medications have been paradoxically associated with increased mortality rates among diabetic patients, particularly in those with ischemic heart disease. ${ }^{8-12}$

Sodium-glucose co-transporter type 2 (SGLT2) inhibitors are a novel medication class that promotes glucose excretion in the kidney. ${ }^{13}$ SGLT2 inhibitors not only improve fasting plasma glucose (FPG) but reduce absolute A1c levels. They also induce blood pressure reductions and weight loss. ${ }^{13,14}$ Moreover, SGLT2 inhibitors have shown to improve cardiovascular and renal outcomes along with all-cause mortality in diabetic patients. ${ }^{4}$ The mechanisms by which SGLT2 inhibitors promote such benefits are not fully understood. However, based on recent preclinical and clinical data, they could play an important role in the stabilization of cellular metabolic stress, glucose-mediated toxicity, and inflammation in key organs such as the kidneys and the heart. ${ }^{15,16}$ This review provides an overview of most relevant findings derived from recent randomized clinical trials and provides a comprehensive review of the mechanistic effects of these medications in the pathophysiology of T2DM and the cardiorenal axis; summarized in Table 1.

\section{Sodium-Glucose Co-Transporters: Physiology and Expression Regulation}

Sodium-glucose co-transporters 2 are apical integral membrane-bound proteins that transport sodium and glucose in an equimolar fashion in the S1 and S2 segments of the proximal tubule. ${ }^{13}$ SGLT2 proteins are part of the SGLT family that include six different isoforms (SGLT1-6) with 
different substrate specificity and tissue localization. ${ }^{17}$ While SGLT2 is predominantly expressed in the kidney, SGLT1 is mainly expressed in the intestine, and in a lesser amount in the S3 segment of the proximal tubules. SGLT2 is responsible for $90 \%$ of the reabsorption of freely filtered glucose. In healthy individuals, approximately $180 \mathrm{~g}$ of glucose is filtered every day assuming that the glomerular filtration barrier is intact. ${ }^{13,17}$ Tubular glucose reabsorption increases linearly with increasing serum glucose levels until the SGLT transport system becomes saturated. Maximal glucose tubular transport (Tmg) is approximately $375 \mathrm{mg} / \mathrm{min}$ in adults; which correlates with $300 \mathrm{mg}$ of glucose per day. ${ }^{18}$ Under physiological conditions, virtually no glucose is detected in urine due to the action of SGLT2 and minor contribution of SGLT1. Furthermore, The activity of SGLT2 increases in the early stages of diabetes via renal tubular growth, hyperplasia, and hypertrophy of the proximal tubules. ${ }^{19,20}$

Asides from SGLT1 and SGLT2, two other proteins located in the basolateral side of the renal epithelial cells are important in glucose transport: the ATP-dependent $\mathrm{Na}$ $+/ \mathrm{K}+$ pump and glucose transporter 2 (GLUT2). ${ }^{13,17}$ High intracellular sodium concentration due to glucose uptake stimulates the ATP-dependent $\mathrm{Na}+\mathrm{K}+$ pump which actively transports sodium back to the circulation while glucose transport is facilitated by gradient-driven GLUT2 in the proximal tubules and GLUT1 in the distal portions of the nephron. ${ }^{21}$ Proximal tubule reabsorption of glucose as well as sodium reabsorption (65\% of filtered sodium) reduce the delivery of sodium and chloride to the macula densa, thus activating the tubuloglomerular feedback (TGF). This mechanism in turn decreases the tone of the renal afferent arteriole; thereby increasing blood renal flow and the single nephron glomerular filtration rate. ${ }^{22}$ Also, the decreased delivery of fluid to the distal portions of the nephron lowers the tubular back pressure in Bowman space. This increases the effective glomerular filtration pressure; accounting for up to $50 \%$ of diabetic hyperfiltration. $^{23}$

The mechanisms that govern the regulation and expression of SGLT2 in diabetes mellitus are yet to be elucidated. Studies in mice have shown that SGLT2 inhibition increases renal expression of SGLT1, suggesting that a decreased glucose delivery to the proximal tubules may trigger a compensatory mechanism to ensure glucose homeostasis. ${ }^{19}$ Ghezzi et $\mathrm{al}^{23}$ demonstrated that glucose transport through SGLT2 is upregulated by protein kinase $\mathrm{A}$ and protein kinase $\mathrm{C}$ in human embryonic kidney
(HEK) 293T cells. In this study, insulin was found to induce a $250 \%$ increase in SGLT2 transport and a marginal reduction of the glucose transport through SGLT1. The authors suspected that these findings correlated with glycemic changes when moving from a fasting state to fed state after a carbohydrate-rich diet. Likewise, studies in cultured human proximal tubular cells by Nakamura et $\mathrm{al}^{24}$ demonstrated that insulin evoked the production of reactive oxygen species (ROS) in cultured proximal tubular cells; an effect that correlated with the upregulation of SGLT2 proteins and increased glucose uptake. Furthermore, they also found that glucose uptake could be markedly blocked by the administration of the antioxidant $\mathrm{N}$-acetyl cysteine; suggesting that ROS production could be a mechanism of insulin to increase glucose uptake in renal epithelial cells. While hyperglycemia and advanced glycation end products (AGEs) have been implicated in the production of ROS previously, ${ }^{25,26}$ studies have failed to show an increase in the expression of SGLT2, supporting a mechanism directly linked to insulin stimulation. Interestingly, SGLT2 can also be upregulated in hypoinsulinemic states as demonstrated in T1DM Akita mice; ${ }^{20,27}$ questioning the argument that the expression of SGLT2 is increased in DM2 at the expense of insulin activity. Alternatively, mechanisms mediated by angioten$\sin$ II AT1 receptors, hepatocyte nuclear factor HNF-1 $\alpha$, and pro-inflammatory cytokines such as IL-6 and TNF- $\alpha$ (autocrine regulation) have been found to upregulate SGLT2 proteins and could explain increased SGLT2 activity in T1DM. ${ }^{28-30}$

\section{SGLT2 Inhibitors and Dual SGLT I/2 Inhibition: Pharmacological Characteristics and Clinical Data}

Dapagliflozin was the first selective SGLT2 inhibitor approved for the treatment of T2DM in 2012. It was followed by the approval of other commercially available SGLT2 inhibitors such as canagliflozin and empagliflozin. $^{31}$ The selectivity of dapagliflozin for SGLT2 is $>1400$-fold greater than that for SGLT1 and it does not inhibit other glucose transporters in peripheral tissues. Dapagliflozin is administered orally in doses of 5-10 mg with peak plasma concentrations in 1.5-2 hours in adults in the fasted state, whereas high-fat meals decrease the peak plasma concentration by approximately $50 \%{ }^{32}$ The oral availability of dapagliflozin after a dose of $10 \mathrm{mg}$ is approximately $78 \%$. It is a protein-bound 
compound $(91 \%)$ with a relatively large volume of distribution at $118 \mathrm{~L}$. Similar to other SGLT2 inhibitors, dapagliflozin has a prolonged half-life (12.9 hours), allowing daily administration. ${ }^{14,32}$ After hepatic and renal glucuronidation via UGT1A9, dapagliflozin is inactivated and eliminated largely through the urine $(75 \%)$ with a minor elimination in bile and feces. The plasma concentration of dapagliflozin may increase in cases of severe liver disease (Child-Pugh C) and advanced kidney disease. ${ }^{31}$ Dose reduction is recommended in patients with liver dysfunction. Furthermore, the use of dapagliflozin has been limited in clinical practice among patients with estimated glomerular filtration rate (eGFR) $<60 \mathrm{~mL} / \mathrm{min} / 1.73 \mathrm{~m}^{2}$ and has been avoided in patients with persistently low eGFR $<45 \mathrm{~mL} / \mathrm{min} / 1.73 \mathrm{~m}^{2.33}$

The effect of dapagliflozin and other SGLT2 inhibitors is dose-dependent and relies on the glomerular filtration rate. Therefore, an attenuation of their effects is expected in patients with impaired kidney function. However, the recently published large randomized placebo-controlled clinical trial "Canagliflozin and Renal Events in Diabetes with Established Nephropathy Clinical Evaluation" (CREDENCE) $)^{34}$ included 1308 (59\%) patients with moderate to severe kidney disease (eGFR $<60 \mathrm{~mL} / \mathrm{min} /$ $\left.1.73 \mathrm{~m}^{2}\right)$ in the treatment arm. Reportedly, only 13 patients $(0.6 \%)$ in the treatment group and 19 patients $(0.9 \%)$ in the placebo group experienced problems related with safety and tolerability that prompted early discontinuation of the therapy. Likewise, serious adverse effects including fractures, amputation, pancreatitis, among others were comparable in patients with reduced eGFR in both groups. Thus, results from CREDENCE suggest that patients with advanced kidney disease could be candidates for therapy with canagliflozin and other SGLT2 inhibitors with a good safety profile. This is particularly important since patients with advanced kidney disease are at the highest risk for secondary complications including heart disease, need for renal replacement therapy, and all-cause mortality. ${ }^{22}$

The safety profile of several SGLT2 inhibitors is under investigation for their use in patients with T1DM due to the potential risk of diabetic ketoacidosis and urinary tract infections in this population. Dual SGLT1/2 inhibitors such as sotagliflozin offer a new therapeutic option in T1DM. Sotagliflozin can effectively block SGLT1 with a high affinity $(\mathrm{Km}=\sim 0.5 \mathrm{mM})$ and reduces the absorption of glucose and galactose in the proximal intestine, improves postprandial glycemic control, and can further increase renal tubular excretion of glucose by simultaneously blocking SGLT2 and SGLT1 receptors. ${ }^{35,36}$ Sotagliflozin is extensively plasma protein bound (>93\%), and at doses of $400 \mathrm{mg}$ exhibits local inhibition of SGLT1 as opposed of systemic blockade. The clinical utility of sotagliflozin is related to reduced postprandial glucose load leading to reduced correctional insulin doses in T1DM patients and a potential lower incidence of

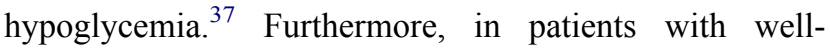
controlled T1DM (A1c < 7.3\%), the contribution of postprandial hyperglycemia to A1c levels has been found to be as high as $70 \%$ whereas in those with poor glycemic control, its contribution was substantially less $(30 \%){ }^{35}$ Therefore, the effects of sotagliflozin on postprandial hyperglycemia are expected to provide the greatest benefit in patients with A1c closer to the lower range. This contrasts with SGLT2 inhibitors, which predominantly optimize fasting plasma glucose, independent of meals. Compared to SGLT2 inhibitors, GLP-1 offers similar cardiovascular and all-cause mortality benefits but with less incidence of adverse events as reported recently. ${ }^{38}$

\section{Clinical Trials on SGLT2 Inhibitors, Cardiovascular and Renal Outcomes: Current Data}

In 2008, the FDA guidance mandated the evaluation of the effect of new antidiabetic therapy in cardiovascular outcome trials (CVOT). Three clinical trials including SGLT2 inhibitors and cardiovascular-related outcomes in longstanding T2DM patients with established or at risk of atherosclerotic heart disease have been conducted: EMPAREG OUTCOME,${ }^{39}$ CANVAS,${ }^{40}$ and Dapagliflozin Effect on Cardiovascular Events-Thrombolysis in Myocardial Infarction 58 (DECLARE-TIMI-58). ${ }^{41}$ However, data exploring primarily renal outcomes in DKD is scant. CREDENCE was the first trial which included 2202 patients with CKD and eGFR from 30 to $90 \mathrm{~mL} / \mathrm{min} /$ $1.73 \mathrm{~m}^{2}$ and albuminuria defined as urinary albumin-tocreatinine ratio $>300-5000 \mathrm{mg} / \mathrm{g}$. CREDENCE had the predetermined goal to study the cardiovascular and renal effect of canagliflozin in those with eGFR $<60 \mathrm{~mL} / \mathrm{min} /$ $1.73 \mathrm{~m}^{2}$ (60\% of the study population). ${ }^{34}$ Characteristics of the study population, inclusion criteria, and specific cardiovascular and renal outcomes are provided in Table 2.

Dapagliflozin safety and benefits on major adverse cardiovascular events (MACE) were assessed in the DECLARE-TIMI-58, including patients with and without 
Table 2 Summary of Clinical Trials Studying SGLT2 Inhibitors in Cardiovascular and Renal Outcomes

\begin{tabular}{|c|c|c|c|c|}
\hline & DECLARE-TIMI 58 & $\begin{array}{l}\text { EMPA-REG } \\
\text { OUTCOME }\end{array}$ & $\begin{array}{l}\text { CANVAS } \\
\text { Program }\end{array}$ & CREDENCE \\
\hline Intervention & Dapagliflozin & Empagliflozin & Canagliflozin & Canagliflozin \\
\hline $\begin{array}{l}\text { Dose } \\
\text { Number of participants } \\
\text { Mean age (years) } \\
\text { Follow up (years) } \\
\text { Established CV disease (\%) } \\
\text { RAAS inhibitors (\%) } \\
\text { eGFR inclusion criteria }\end{array}$ & $\begin{array}{l}10 \mathrm{mg} \\
17,160 \\
63.9 \\
4.2 \\
41 \\
81.3 \\
\mathrm{CrCl} \geq 60 \mathrm{~mL} / \mathrm{min} \text { (Cockcroft- } \\
\text { Gault) }\end{array}$ & $\begin{array}{l}10 \mathrm{mg}, 25 \mathrm{mg} \\
7020 \\
63.1 \\
3.1 \\
41 \\
81 \\
\geq 30 \text { (MDRD) }\end{array}$ & $\begin{array}{l}100 \mathrm{mg}, 300 \mathrm{mg} \\
10,142 \\
63.3 \\
2.4 \\
66 \\
80.2 \\
\geq 30 \text { (MDRD) }\end{array}$ & $\begin{array}{l}100 \mathrm{mg} \\
4401 \\
63 \\
2.6 \\
99 \\
99.9 \\
30-90 \text { (CKD-EPI) }\end{array}$ \\
\hline $\begin{array}{l}\text { Baseline eGFR }(\mathrm{mL} / \mathrm{min} / \\
\left.1.73 \mathrm{~m}^{2}\right) \\
\geq 90 \\
60-90 \\
45-60 \\
<45\end{array}$ & $\begin{array}{l}8162(47.6 \%) \\
7732(45.1 \%) \\
1265(7.4 \%) \\
554(5.5 \%)\end{array}$ & $\begin{array}{l}1538(21.9 \%) \\
366 \mid(52.2 \%) \\
1249(17.8 \%) \\
570(8.1 \%)\end{array}$ & $\begin{array}{l}2476(24.4 \%) \\
5625(55.5 \%) \\
1485(14.6 \%) \\
554(5.5 \%)\end{array}$ & $\begin{array}{l}0 \\
1809(41 \%) \\
1279(29.1 \%) \\
1313(29.8 \%)\end{array}$ \\
\hline UACR criteria (mg/g) & None & None & None & $>300-5000$ \\
\hline $\begin{array}{l}\text { Baseline UACR subgroup } \\
\text { ( } \mathrm{mg} / \mathrm{g}) \\
\quad<30 \\
30-300 \\
>300\end{array}$ & $\begin{array}{l}11,644(67.9 \%) \\
4030(23.5 \%) \\
1169(6.8 \%)\end{array}$ & $\begin{array}{l}4171(59.4 \%) \\
2013(28.7 \%) \\
769(11 \%)\end{array}$ & $\begin{array}{l}7007(69.1 \%) \\
2266(22.3 \%) \\
760(7.5 \%)\end{array}$ & $\begin{array}{l}0 \\
0 \\
4401(100 \%)\end{array}$ \\
\hline $\begin{array}{l}\text { Primary outcome } \\
\text { (HR [95\% Cl]) }\end{array}$ & $\begin{array}{l}\text { 3-point MACE } \\
0.93(0.84-1.03)\end{array}$ & $\begin{array}{l}\text { 3-point MACE } \\
0.86(0.74-0.99)\end{array}$ & $\begin{array}{l}\text { 3-point MACE } \\
0.86(0.75-0.97)\end{array}$ & $\begin{array}{l}\text { Primary composite Kidney and CV } \\
\text { outcome* } \\
0.70(0.59-0.82)\end{array}$ \\
\hline $\begin{array}{l}\text { Sec. outcomes } \\
\text { (HR }[95 \% \mathrm{Cl}]) \\
\text { CV death } \\
\text { MI } \\
\text { Stroke } \\
\text { Hospitalization HF }\end{array}$ & $\begin{array}{l}0.98(0.82-1.17) \\
0.89(0.77-1.01) \\
1.01(0.84-1.21) \\
0.73(0.61-0.88)\end{array}$ & $\begin{array}{l}0.62(0.49-0.77) \\
0.87(0.70-1.09) \\
1.18(0.89-1.56) \\
0.65(0.50-0.85)\end{array}$ & $\begin{array}{l}0.87(0.72-1.06) \\
0.89(0.73-1.09) \\
0.87(0.69-1.09) \\
0.67(0.52-0.87)\end{array}$ & $\begin{array}{l}0.69(0.57-0.83) \\
0.80(0.67-0.95)^{* *} \\
0.61(0.47-0.80)\end{array}$ \\
\hline $\begin{array}{l}\text { All-cause mortality } \\
\text { (HR }[95 \% \mathrm{Cl}])\end{array}$ & $0.93(0.82-1.04)$ & $0.68(0.57-0.82)$ & $0.87(0.74-1.01)$ & $0.83(0.68-1.02)$ \\
\hline
\end{tabular}

Notes: *ESRD (dialysis, transplantation, or sustained eGFR $<15 \mathrm{~mL} / \mathrm{min} / \mathrm{I} .73 \mathrm{~m}^{2}$ ), doubling of the serum creatinine, or death from renal or CV causes. $* * C V$ death, $\mathrm{Ml}$ or stroke: 38.7 vs 48.7/I000 P-Y (HR 0.80; 95\% Cl 0.67-0.95; P=0.0I), NNT=40 (23-165).

Abbreviations: CV, cardiovascular; RAAS, renin-angiotensin-aldosterone system; eGFR, estimated glomerular filtration rate; UACR, urine albumin-creatinine ratio; HR, hazard ratio; $\mathrm{MI}$, myocardial infarction; $\mathrm{HF}$, heart failure; $\mathrm{Cl}$, confidence interval.

cardiovascular disease $(60 \%) .{ }^{41}$ After a median follow up of 4.2 years, the authors found that dapagliflozin was superior to placebo in improving serum glucose levels and noninferior (but not superior) to reduce MACE in T2DM $[8.8 \%$ vs $9.4 \%$, hazard ratio (HR) $0.93,95 \%$ confidence interval (CI) $0.84-1.03$; $\mathrm{P}<0.001$ for non-inferiority and $\mathrm{P}=0.17$ for superiority]. Compared to placebo, dapagliflozin was also found to reduce HF hospitalizations $(2.5 \%$ vs
$3.3 \%, \mathrm{P}<0.05$ ), and $>40 \%$ reduction in eGFR, end-stage kidney disease (ESKD), or death due to renal or cardiovascular causes $(4.3 \%$ vs $5.6 \%, \mathrm{p}<0.05)$. On further analysis, when patients were stratified by history of myocardial infarction (MI), dapagliflozin was found to reduce the relative risk of MACE by $16 \%$ and the absolute risk by $2.6 \%$ in patients with prior MI (15.2\% vs $17.8 \%$, HR 0.84, 95\% CI $0.72-0.99 ; \mathrm{p}=0.039) .{ }^{42}$ Furthermore, there was no 
difference in HF hospitalizations in this sub-study from DECLARE-TIMI-58. Interestingly, when Kato et al. ${ }^{43}$ investigated the effects of dapagliflozin in patients with and without $\mathrm{HF}$ and reduced ejection fraction $(\mathrm{rEF}<45 \%)$, they found that it decreased CV death and HF hospitalizations. These findings were statistically significant in patients with HFrEF (HR 0.62, 95\% CI 0.45-0.86) compared to patients without HFrEF (HR 0.88, 95\% CI 0.76-1.02; p-interaction 0.046 ), despite the low proportion of patients with HFrEF (3.9\% of the study group). These findings could be partially explained by the combined effect of ACEi/ARBs, beta-blockers, and aldosterone blockers in patients HFrEF since SGLT2 inhibitors have been found to exert synergistic cardioprotective effects. ${ }^{44}$ Also, dapagliflozin was found to reduce hospitalization for $\mathrm{HF}$ in both patients with HFrEF (HR 0.64, 95\% CI 0.43-0.95) and without HFrEF (HR 0.76, 95\% CI 0.62-0.92); while it only reduced $\mathrm{CV}$ death in the former group (HR 0.55 , 95\% CI $0.34-0.90){ }^{43}$

The EMPA-REG OUTCOME trial allocated T2DM patients in a $1: 1: 1$ fashion to either treatment with empagliflozin $10 \mathrm{mg}(\mathrm{n}=2345), 25 \mathrm{mg}(\mathrm{n}=2342)$, or matching placebo $(n=2333) .{ }^{39}$ Patients had significant history of atherosclerotic heart disease including MI (47\%), multiple vessel disease (47\%), coronary artery bypass grafting $(25 \%)$, and eGFR $>30 \% \mathrm{~mL} / \mathrm{min} / 1.73 \mathrm{~m}^{2}$. This trial demonstrated that compared to placebo, empagliflozin improved a primary composite endpoint including cardiovascular death, myocardial infarction, or stroke $(10.5 \%$ vs $12.1 \%$, HR 0.86 , 95\% CI $0.74-0.99$; $\mathrm{p}<0.001$ for noninferiority; $\mathrm{p}=0.04$ for superiority). When cardiovascular death, MI or stroke were evaluated individually, empagliflozin was found to improve CV death rates only $(3.7 \%$ vs $5.9 \%, \mathrm{p}<0.001)$. Moreover, empagliflozin improved allcause mortality $(3.8 \%$ vs $5.1 \%, \mathrm{p}<0.01)$ and HF hospitalization $(2.7 \%$ vs $4.1 \%, \mathrm{p}=0.002)$. Likewise, improved renal outcomes such as worsening nephropathy $(12.7 \%$ vs 18.8\%, HR 0.61, 95\% CI 0.53-0.70; p < 0.001), doubling of serum creatinine $(1.5 \%$ vs $2.6 \%, \mathrm{p}<0.001)$ and initiation of RRT $(0.3 \%$ vs $0.6 \%, p=0.04)$ were associated with empagliflozin when compared to placebo. EMPA-REG OUTCOME showed that empagliflozin held promise in the management of T2DM and heart disease especially when the kidney function is affected; even when only modest reductions of A1c $(0.5 \%)$ were noted in the treatment arm. Results for composite renal outcomes were validated in a post hoc sensitivity analysis by Wanner $\mathrm{C}$ et $\mathrm{al}^{45}$ showing that progressive kidney disease ("worsening nephropathy") was less frequent in the empagliflozin group when compared to placebo $(12.7 \%$ vs 18.8\% HR 0.61, 95\% CI 0.53-0.70; P<0.001). Likewise, doubling of serum creatinine level (1.5\%) and initiation of RRT $(0.3 \%)$ occurred in a minority of patients in the treatment arm, accounting for a $44 \%$ and 55\% lower relative risk, respectively. Authors raised caution on these findings since renal benefits seen in this specific study population (high cardiovascular risk) may not be extrapolated to those T2DM patients with a low cardiac disease risk. Similarly, given the underrepresentation of ethnical minorities (African American, Asian, Hispanic, etc.) and patients with extreme body mass indexes (BMI $>35-40)$, additional caution should be considered until further data is available.

The CANVAS Program, ${ }^{40}$ comprising two sister trials (CANVAS and CANVAS-RENAL), was designed to assess the safety and efficacy of canagliflozin and to evaluate the cardiovascular and renal benefits versus the risks associated with this therapy including genitourinary infection, diabetic ketoacidosis, and fracture. ${ }^{40,46}$ The primary outcome was a composite of death from cardiovascular causes, nonfatal MI, or nonfatal stroke, which occurred in 26.9 participants per 1000 patient-years in the canagliflozin group versus 31.5 participants per 1000 patient-years in the placebo group ( $p=0.02$ for superiority, $p<0.001$ for non-inferiority). Renal outcomes including progression of albuminuria occurred less frequently among participants in the treatment arm (89.4 participants per 1000 patientyears) compared to those assigned to placebo (128.7 participants per 1000 patient-years); with a hazard ratio of 0.73 (95\% C I, 0.67-0.79). Furthermore, the composite outcome of sustained $40 \%$ reduction in eGFR, RRT need, or death from renal causes occurred less frequently in the treatment group (5.5 participants per 1000 patient-years) compared to placebo (9.0 participants per 1000 patientyears, HR 0.60, 95\% CI: 0.47-0.77). Additionally, the benefit of canagliflozin appeared to be similar for patients with $\mathrm{HFrEF}$ and $\mathrm{HF}$ with preserved ejection fraction (HFpEF). Importantly, the authors found that canagliflozin was associated with a higher risk of amputation of toes, feet, or legs especially in those with underlying peripheral artery disease or history of amputation; a link that was no evidenced in EMPA-REG OUTCOME. The benefit of SGLT2 inhibitors in heart failure has been already acknowledged by some societal guidelines including the Heart Failure Association of the European Association of Cardiology. $^{47}$ 
DECLARE-TIMI-58, EMPA-REG OUTCOME, and CANVAS explored cardiovascular benefits associated with SGLT2 inhibitors in large groups of patients with variable degree of DKD (most with GFR $>60 \mathrm{~mL} / \mathrm{min} /$ $1.73 \mathrm{~m}^{2}$ ). However, the question if SGLT2 inhibitors were effective and safe for patients with moderate to severe kidney function impairment was finally addressed by the CREDENCE trial. ${ }^{34}$ CREDENCE randomized patients with T2DM and eGFR from 30 to $60 \mathrm{~mL} / \mathrm{min} / 1.73 \mathrm{~m}^{2}$ to either canagliflozin $(\mathrm{N}=2,202)$ or placebo $(\mathrm{N}=2199)$. The primary outcome was a composite of 1) ESKD (dialysis for at least 30 days, kidney transplantation, or persistent eGFR $<15 \mathrm{~mL} / \mathrm{min} / 1.73 \mathrm{~m}^{2}$ for at least 30 days) and 2) persistent doubling serum creatinine level or death from renal or cardiovascular disease. CREDENCE was prematurely stopped after 2.6 years due to the achievement of the primary composite endpoint in the interim analysis. Canagliflozin was found to decrease ESKD, doubling of serum creatinine, and renal or cardiovascular death compared to placebo (43.2 vs 61.2 per 1000 patient-years; $\mathrm{P}<$ 0.001). Furthermore, renoprotective effects were seen even in those with more advanced kidney disease and eGFR of $30-45 \mathrm{~mL} / \mathrm{min} / 1.73 \mathrm{~m}^{2}$ (0.75 HR, 95\% CI 0.59-0.95). This observation is particularly important since these patients are at increased risk of complications including rapid progression of $\mathrm{DKD}$, and kidney replacement therapy. Furthermore, the rates of AKI were comparable among both groups, suggesting that mild decrements of GFR are expected at the beginning of treatment. However, these changes are compensatedby the so expected "renal protection." Patients in the treatment arm presented a slower decline in eGFR $\left(1.52 \mathrm{~mL} / \mathrm{min} / 1.73 \mathrm{~m}^{2}\right.$ per year) when compared to placebo. Moreover, benefits were obtained in association with RAAS blockade therapy, which is another medication that can induce mild GFR reduction that are not necessarily associated with kidney damage. This finding supports the synergistic effect exerted by these medications and together could explain such overwhelming benefits in DKD. In addition, the protective kidney and cardiovascular effects were seen in patients with moderate-severe kidney impairment without a statistically significant increase in the rate of amputations in the treatment group compared to placebo $(3.2 \%$ vs 2.9\%, HR 1.11, 95\% CI: 0.79-1.56).

Among the secondary outcomes, CREDENCE demonstrated that canagliflozin was associated with a lower risk of cardiovascular death or hospitalization for heart failure (HR 0.69; 95\% CI, 0.57-0.83; P < 0.001), cardiovascular death, MI, or stroke (HR 0.80, 95\% CI: 0.67-0.95; P = 0.01 ) and hospitalization for heart failure (HR: 0.61; $95 \%$ CI, 0.47 to $0.80 ; \mathrm{P}<0.001)$. The hazard ratio for death from any cause was $0.83(95 \% \mathrm{CI}, 0.68$ to 1.02$)$. In contrast to the primary analysis of DECLARE-TIMI-58, ${ }^{41}$ where dapagliflozin did not reduce the rate of $\mathrm{MACE}$ in the treatment group compared to placebo $(8.8 \%$ vs $9.4 \%$, HR 0.9, 95\% CI: 0.84-1.03; P = 0.17), CREDENCE revealed a $20 \%$ reduction in atherosclerotic heart disease (MI, nonfatal stroke) and cardiovascular death in agreement with the results from CANVAS. It is unclear whether these differences are explained by patient-specific characteristics and less likely by a difference in the medication used. However, it is possible the burden of advanced kidney disease, which is a major contributor for cardiovascular disease, is significantly modified by SGLT2 inhibitors as shown in CREDENCE. CREDENCE provides additional information about the putative true role of SGLT2 inhibitor therapy in patients with advanced kidney disease. In CREDENCE, canagliflozin exerted greater reductions of A1c levels when compared to those in the placebo group during the initial 12 months of enrollment (mean difference: $-0.25 \%$, CI $-0.31 \%-0.20 \%$ ). However, this effect seemed to decrease posteriorly with an end of the study difference of $-0.11 \%(95 \% \mathrm{CI}-0.28-0.06, \mathrm{P}>0.05)$. This finding requires careful interpretation since glucose lowering effects of SGLT2 inhibitors depend on GFR and renal glucose excretion; compromising A1c reductions. Nonetheless, the benefits in the cardiorenal axis persisted and were clinically significant in patients with low GFR, suggesting that 1) SGLT2 inhibitors should be continued despite marginal glucose lowering effects in patients with CKD, 2) serum glucose targets should not mandate the prescription of SGLT2 inhibitors in DKD and 3) it is possible that additional off-target effects contribute to improved outcomes in renal patients.

Most recently, Dapagliflozin and Prevention of Adverse Outcomes in Chronic Kidney Disease (DAPACKD) explored the composite of sustained decline in the eGFR of at least $50 \%$, ESKD or death from renal or cardiovascular causes. ${ }^{48}$ Among 4304 adult participants with albumin-to-creatinine ratio $>200 \mathrm{mg} / \mathrm{g}$ and eGFR between 25 and $75 \mathrm{~mL} / \mathrm{min} / 1.73 \mathrm{~m}^{2}$, there were 197 primary endpoint events with dapagliflozin and 312 with placebo. The HR for the primary endpoint was 0.61 (95\% CI 0.51-0.72; $\mathrm{P}<0.001)$. These results were consistent in patients with and without DM2. Furthermore, Dapagliflozin provided cardiorenal benefits compared to 
placebo as evidenced in the secondary endpoints including 1) worsening renal function or death from kidney failure (HR: $0.56,95 \%$ CI $0.45-0.68$; $\mathrm{P}<0.001$ ), 2) hospitalization for heart failure or cardiovascular death (HR: $0.71,95 \%$ CI $0.55-0.92 ; \mathrm{P}=0.0089$ ) and 3) all-cause mortality (HR: $0.69,95 \%$ CI $0.53-0.88$; $\mathrm{P}=0.0035$ ). DAPACKD provides further insight in the role of SGLT2 inhibitors in the progression of CKD and supports their use to avoid the onset of cardiovascular and kidney-related complications among CKD patients. Due to the complexity of the mechanisms associated with SGLT2 inhibitors, we provide a comprehensive review of them based on their effect on multiple organs in the following lines.

\section{Mechanism of Action: Renal and Extra-Renal Effects}

Sodium-glucose co-transporters 2 blockade increases the urinary excretion of glucose and sodium, decreasing glucose concentrations and products of glucose metabolism in the renal epithelial cells. Increased delivery of sodium to the macula densa increases the tone in the afferent renal artery via TGF, ultimately reducing the intraglomerular pressure and mitigating the urinary excretion of albumin. Glucose is still absorbed downstream in the nephron through SGLT1 and other putative glucose transporters including GLUT9, which exchanges glucose for uric acid. ${ }^{13}$ The contribution of the latter transporter to overall glucose reabsorption is unknown. However, serum uric acid levels have been shown to decrease with SGLT2 inhibitors in clinical studies. Therefore, net glucose excretion is less that it would be expected after complete SGLT2 blockade; being approximately $25-65 \%$ of the filtered glucose load. ${ }^{22}$

The natriuretic effects of SGLT2 are transient, probably due to compensatory mechanisms that increase $\mathrm{Na}+$ reabsorption such as the renin-angiotensin-aldosterone system (RAAS). Despite this, a negative $\mathrm{Na}^{+}$balance is eventually reached, and a new steady state is set at a reduced level of total body $\mathrm{Na}^{+}$composition. SGLT2 inhibitors differ from loop diuretics and thiazide diuretics in that they do not lead to reflex sympathetic nervous system activation, thereby reducing neurohormonal changes and cellular stress. ${ }^{49}$ Osmotic diuresis related with glucose excretion and natriuresis have been hypothesized to explain rapid blood pressure changes induced with SGLT2 inhibitors. However, there is conflicting evidence regarding the duration of plasma volume reductions. In a small placebo-controlled study, Sha et $\mathrm{al}^{50}$ demonstrated that while the effect of canagliflozin on glycosuria and FPG was maintained by 12 weeks of therapy, plasma volume reductions were transient and only lasted 1 week. In contrast, Lambers Heerspink $\mathrm{HJ}$ et $\mathrm{al}^{51}$ demonstrated that blood pressure reductions were persistent after 12 weeks of therapy with dapagliflozin. Interestingly, the authors noted that the median reduction in plasma volume was offset by a comparable increase in the hematocrit concentration $(2.2 \%)$ in the treatment group which persisted even when the effect of volume contraction subsided. $^{51}$ Moreover, SGLT2 inhibitors induce a negative caloric balance responsible for $2-3 \%$ weight loss. This is less than expected for the degree of glycosuria obtained with this therapy and could be related to compensatory mechanisms such as energy preservation and hyperphagia; demonstrated in animal models. ${ }^{52}$

\section{Emerging Evidence on the Effect of SGLT2 Inhibitors on Glucose-Mediated Cellular Toxicity, Energetic Balance and Modulation of Inflammation in Renal and Cardiac Tissue}

\section{Glucose-Mediated Cellular Toxicity: Clinical and Biochemical Appraisal}

Chronic hyperglycemia induces tissue damage via major metabolic pathways. Hyperglycemia increases glucose oxidation and the generation of superoxide as a part of the process of electron transmission in the mitochondrial respiratory chain. Alternatively, the excess of glucose can also be metabolized to sorbitol through the polyol pathway, a process that results in NADPH depletion thereby limiting the synthesis of the natural antioxidant glutathione which renders cells more prone to oxidative damage., ${ }^{4,53}$

Furthermore, high glucose concentrations in the podocyte activate the mammalian target of rapamycin (mTOR) signaling pathway via ErK1/2, resulting in energy consumption, endoplasmic reticulum stress, and apoptosis of these cells. ${ }^{54}$ Moreover, excessive intracellular glucose is metabolized to fructose-6-phosphate; a compound that can undergo further modifications in the hexosamine pathway and result in additional ROS generation and toxic byproducts. Also, hyperglycemia promotes B-O-linkage of $\mathrm{N}$-acetyl glucosamine (O-GlcNAc) which can induce posttranslational modifications including the non-enzymatic 
glycation of nuclear and plasmatic proteins and lipids; altering the cellular structure. ${ }^{53}$ Furthermore, AGEs play an important role in the progression of atherosclerosis through oxidative stress and pro-inflammatory responses through the interaction with RAGE (receptor of AGE); which is highly expressed in the kidneys, heart, and vasculature. ${ }^{55}$ These paths have been implicated in the overexpression of cellular signal pathways associated with cellular stress and fibrosis such as TGF-B1 and makers of cellular injury and necrosis including IL- $8 .{ }^{56}$

\section{Heart Failure, Diabetic Cardiomyopathy and SGLT2}

From a physiological perspective, myocardial cells are flexible in the source of metabolic fuel used. They can obtain energy from glucose, free fatty acids (FFAs), lactic acid, ketones, etc. However, in the setting of hyperglycemia, insulin resistance, and hypertriglyceridemia, the myocardium predominantly uses FFAs and has reduced the ability to utilize glycolytic pathways. This is accompanied by impaired oxidative phosphorylation and mitochondrial proton leak; leading to ROS generation. ${ }^{57,58}$ Due to limited antioxidant capacity in the myocardium, increased ROS production leads to nitric oxide (NO) consumption, impaired cardiac relaxation, and progressive myocardial stiffness; hallmarks of diabetic cardiomyopathy. Moreover, in the failing heart, the capacity of the myocardium to utilize FFAs eventually becomes affected, relying on ketone bodies as an alternative energy source..$^{59,60}$

SGLT2 inhibitors can shift the metabolism from glucose to fatty acid oxidation and increase plasma concentrations of ketones. ${ }^{61}$ This fuel selection serves to a more efficient utilization of oxygen and to improve the mitochondrial performance; restoring myocardial function in diabetes. ${ }^{62}$ Although ketone levels were not measured in the 'Empagliflozin Cardiovascular Outcome Event Trial in Type 2 Diabetes Mellitus Patients' (EMPA-REG OUTCOME) $)^{39}$ or in CANVAS, ${ }^{40}$ it is possible they have decreased heart failure admissions by restoring at least at some extent the heart function in these patients. This, in addition to providing the known diuretic and blood pressure controlling effects.

SGLT2 inhibitors block the sodium-hydrogen exchanger (NHE) isoform 1, which is expressed in the myocardium and plays a role in the regulation of intracellular pH. ${ }^{63}$ NHE1 is upregulated in heart failure, which leads to an increase in intracellular $\mathrm{Ca}^{2+}$ concentration, via increased uptake of $\mathrm{Na}^{+}$. As a result, calcium dependentcalcineurin signaling pathway is activated resulting in myocardial cell death, hypertrophy, and tissue damage. ${ }^{64,65}$ Additional studies have demonstrated that empagliflozin can block such effects mediated by NH1 ${ }^{66,67}$ Long-term suppression of NHE1 in animals has demonstrated to reduce oxidative stress and thus, myocardial fibrosis and left ventricular remodeling. ${ }^{68}$ Empagliflozin has been shown to reduce myocardial oxidative stress and fibrosis in DM murine models after 8 weeks of treatment. ${ }^{69}$ Furthermore, Lee TM et al ${ }^{70}$ demonstrated that dapagliflozin reduces collagen synthesis in rats by inhibiting myofibroblast differentiation via STAT3 signaling following acute MI. Dapagliflozin appears to ameliorate some of the toxic effects of angiotensin II-stressed $\mathrm{db} / \mathrm{db}$ mice by decreasing TNF-alpha, and Toll-like receptor 4 (TLR4). Dapagliflozin also inhibits the expression of voltage-dependent L-type calcium channel (CACNA1C), the sodium-calcium exchanger (NCX), and NHE membrane transporters; independent of glucose concentration. ${ }^{71}$ Collectively, this complex array of effects could result in cardiac protection, improved performance, and beneficial outcomes in patients with and without T2DM; as seen in EMPA-REG OUTCOME and the recent DAPA-HF. ${ }^{72}$

\section{Myocardial Ischemia, Fibrosis and SGLT2 Inhibitors}

There is no evidence of SGLT2 expression in the myocardium. However, SGLT1 receptors are present in lower levels in the healthy myocardium and can be upregulated in pathological conditions such as ischemia and heart failure ${ }^{73,74}$ It is unclear whether SGLT1 exerts protective or deleterious effects in the heart. While SGLT1 could facilitate glucose uptake in the cardiomyocyte and serve as an alternative energy source in hypoxic states through anaerobic glycolysis, SGLT1 can also mediate glycogen storage and hypertrophic cardiomyopathy, thereby impairing the energety balance of the myocyte. $\mathrm{Li} \mathrm{Z}$ et $\mathrm{al}^{75}$ demonstrated that mice with cardiomyocyte-specific knockdown of SGLT1 (TGSGLT1-DOWN) experience milder forms of myocardial $\mathrm{I} / \mathrm{R}$ injury in vivo, ex-vivo, and in-vitro in a multistep series of experiments. Additionally, the authors found that SGLT1 activation is mediated via AMPK and ERK by binding of Sp1 and hepatocyte nuclear factor 1 to the Slc5a1 promoter, intensifying SGLT1 mRNA stability through Human Antigen 
$\mathrm{R}$, and enhancing its translocation from intracellular vesicles to the sarcolemma. ${ }^{75}$ Although SGLT2 inhibitors have a higher affinity for SGLT2 receptors compared to SGLT1, the administration of $10 \mathrm{Mmol} / \mathrm{L}$ of canagliflozin via perfusion protocol (equivalent to human circulating levels of $300 \mathrm{mg}$ canagliflozin) was sufficient to block both SGLT2 and SGLT1 in ex-vivo experiments. ${ }^{76}$ This suggests that at least theoretically, SGLT2 inhibitors could exert some effects in cases of myocardial ischemia. Moreover, Lim VG and colleagues demonstrated that in Zucker Diabetic Fatty (ZDF) and nondiabetic Zucker Lean (ZL) rats, administration of canagliflozin was protective against myocardial infarction and significantly reduced the infarct size. ${ }^{76}$ Compared to chow-fed ZDF rats, ZDF rats exposed to canagliflozin for 4 weeks experienced a statistically significant attenuation of the infarct size from $37 \pm 3 \%$ to $20 \pm 2 \%$ of the area at risk. Likewise, the infarct size decreased from $55 \pm 7 \%$ to $27 \pm 3 \%$ in non-diabetic rats. Interestingly, these benefits were not observed in ex-vivo series of experiments. On the contrary, after a washout period, acute canagliflozin administration did not reduce the infarct size, suggesting its effect is not necessarily related to receptor blockade and is different from ischemia-induced preconditioning. Potential protective mechanisms involved in I/R injury could be JAK-STAT signaling pathways modulation, ${ }^{77}$ AMPK amplification, ${ }^{78,79}$ and/or NHE1 inhibition. ${ }^{80}$

\section{Renal Hemodynamics, Albuminuria and Progressive Kidney Disease}

SGLT2 inhibitors directly reduce glomerular hyperfiltration which is a key mechanism associated with rapid function loss and GFR decline in DKD. In a large prospective cohort of Caucasian patients with T2DM who received guideline-directed medical therapy, Ruggenenti $\mathrm{P}$ et $\mathrm{al}^{81}$ demonstrated that the average GFR decline in patients with DKD was $3 \mathrm{~mL} / \mathrm{min} / 1.73 \mathrm{~m}^{2}$ per year, which is three- to five-fold faster than that reported in the general population. ${ }^{82,83}$ However, long-term GFR decline was found to be independent of severity of albuminuria, or concomitant treatment with ACE inhibitors, suggesting that other mechanisms asides from proteinuria contribute to progressive renal dysfunction. ${ }^{81}$ In addition to increased shear stress on the glomerular capillaries and impairment of podocyte functioning, hyperfiltration per se increases tubular flow and the exposure of the kidney to higher loads of glucose, aminoacids, and other products. ${ }^{84}$ Glucose uptake results in the inhibition of AMPK leading to tubular growth, lipid accumulation, and activation of PKC, mTOR, and TGF- $\beta$ related-pathways. ${ }^{19,85,86}$ These molecular pathways lead to tubular hypertrophy, lipid cytoplasmic inclusions, tubule-interstitial inflammation, hypoxia, and fibrosis. Furthermore, tubular load increases oxygen consumption leading to renal hypoxemia, ROS generation, and fibrosis. Also, since reabsorption of glucose, phosphorus, aminoacids, and other products is coupled to sodium uptake, incremental intracellular concentrations of sodium and stimulation of the ATP-dependent $\mathrm{Na}+/ \mathrm{K}+$ increase the intracellular ADP/ATP ratio. This could limit the energy would otherwise be directed to physiologic functions such as autophagy and tissue repair. ${ }^{87}$ In fact, mathematical modeling of $\mathrm{Na}+$ dynamics and $\mathrm{O} 2$ handling in the diabetic rat predicted that diabetes increase $\mathrm{Na}+$ transport and $\mathrm{Na}+$ transport-dependent oxygen consumption by $50 \%$ and $100 \%$, respectively; highlighting the importance of energy unbalance in the hyperfiltrating kidney. ${ }^{88}$ Furthermore, the increment of intracellular glucose promotes the generation of AGEs which binds with RAGE to induce further oxidative stress. Interestingly, RAGE, TLR-4, and High-mobility group box 1 (HMGB1) are upregulated in renal cells in the context of DKD and promote marked inflammatory changes. ${ }^{89}$ This stresses the connection between cellular glucose toxicity and a pro-inflammatory profile in diabetes mellitus.

A recent study in cultures of primary human renal proximal tubular epithelial cells showed that high glucose per se increases SGLT2 expression and glucose consumption. ROS are overproduced, and GAPDH is inhibited. The intracellular accumulation of glucose due to GAPDH inhibition glycolytic products is diverted into four noxious pathways: the polyol pathway, the hexosamine pathway, the lipid synthesis pathway mediated by PKC activity, and AGEs formation pathway. Eventually, these paths lead to overproduction of TGF- $\beta 1$ and IL-8, as well as to cell necrosis and apoptosis. Dapagliflozin ameliorates all the above cascade of events.

\section{Renal Inflammation, Biomarkers of Kidney Injury and Fibrosis}

In streptozocin-induced diabetic mice, treatment with $10 \mathrm{mg} / \mathrm{kg}$ of empagliflozin during 4 weeks has been found to decrease HMGB1, RAGE, and TLR-4 levels in renal tissue. ${ }^{90}$ These changes were elicited by a reduction in the activity of NF-kB and attenuation of pro- 
inflammatory and pro-fibrotic genes including TGF-B, fibronectin, TNF-alpha, monocyte chemoattractant protein-1 (MCP1), CXCL12, and RANTES. Furthermore, urinary markers of kidney injury such as IL- 6 and $\alpha-1$ acid glycoprotein $(A G P)$ and caspases were also found to be reduced in the empagliflozin group compared to placebo-treated mice. Empagliflozin has also shown to ameliorate renal inflammation by decreasing IL-6 levels, and to reduce glomerular hyperfiltration and albuminuria in the Akita mice model (T1DM). ${ }^{90}$ However, in a separate experiment, empagliflozin reduced tubule-interstitial fibrosis without improving the concentration of urinary markers of tubular damage [including kidney injury molecule-1 (KIM1), neutrophil gelatinase-associated lipocalin (NGAL)] nor albuminuria. ${ }^{91}$ A small placebo-controlled, double-blind, crossover trial found that the effects of dapagliflozin on albuminuria are variable. ${ }^{92}$ However, EMPAREG OUTCOME showed that empagliflozin reduced the progression to macroalbuminuria $(>300 \mathrm{mg} / \mathrm{dL})$ in the treatment group when compared to the placebo $(11.2 \%$ vs $16.2 \%, \mathrm{P}<0.001) .{ }^{39}$ Likewise, the CANVAS trial demonstrated that worsening proteinuria was less frequent among patients in the treatment group (89.4 participants per 1000 patient-years) compared to individuals in the control arm (128.7 participants per 1000 patient-years, $\mathrm{P}<0.05){ }^{40}$ Furthermore, in a post hoc analysis from EMPA-REG OUTCOME, individuals in the treatment group presented lower urinary levels of IL-6, and KIM1 when compared to placebo. ${ }^{93}$

\section{Hypoxia, Erythropoiesis, and Tubular Function Changes}

Hypoxia plays an important role in the development of renal interstitial fibrosis and in the progression to CKD. Preservation of renal oxygenation particularly in the kidney cortex has been proposed to protect kidney function in patients with CKD since it is highly active in its metabolic demands. ${ }^{94}$ Hypoxic microenvironment in the proximal tubules in diabetes arises from metabolic stress associated with high glucose, and increased activity of ATP-dependent $\mathrm{Na}+/ \mathrm{K}+$ pump associated with $\mathrm{Na}+$ uptake, leading to the generation of ROS and tubular cell damage. This could trigger the differentiation of erythropoietin-producing peritubular fibroblasts into myofibroblasts responsible of the production of fibrotic tissue. $^{95}$ In patients with diabetes, erythropoietin levels have been found to be decreased even when serum creatinine levels and GFR are within range. ${ }^{96}$ Furthermore, reductions in the production of erythropoietin have been correlated with increasing A1c levels. ${ }^{97}$ Treatment with empagliflozin and canagliflozin increases hematocrit by $2-4 \%$ compared to placebo and is noticed to appear early after starting therapy. ${ }^{51}$ While part of the hematocrit increment could be related to the diuretic effect associated with SGLT2 inhibitors, additional studies have demonstrated that the effect of plasma volume reduction was transient (2-12 weeks) while hematocrit increments were sustained; indicating increased erythropoiesis. ${ }^{51,98}$ Although speculative, it is possible that myofibroblasts could reverse back to erythropoietin-producing fibroblast at least to some extent, a process that may involve Hypoxia-inducible factor 2 (HIF-2) activation. ${ }^{87,99-101}$ Furthermore, an increment of erythropoietin could also contribute tissue protection through the upregulation of the renal mRNA expression of heme oxygenase-1, a HIF-1 induced tissue protective gene, as demonstrated in SGLT2 knockout rats. ${ }^{27}$ Likewise, this effect could not only benefit the kidney but also extend to tissues susceptible to hypoxia such as the myocardium. The effect of SGLT2 inhibitors on EPO production and fibroblast profile modulation deserves further investigation as this could be a novel target to reduce heart failure and ESKD.

Diabetic rats exhibit low concentrations of oxygen in the renal cortex and medulla. Acute administration of phlorizin, which inhibits both SGLT2 and SGLT1, normalizes oxygen tension in the cortex and causes hypoxia in the renal medulla of anaesthetized diabetic rats. ${ }^{87}$ From a physiologic perspective, the medulla is a site vulnerable to hypoxic injury, which can further increase in the setting of SGLT2 inhibition. Also, SGLT2 inhibitors increase the delivery of glucose in O2-restricted areas such as the outer and inner medulla that are dependent on GLUT9; thereby posing tubular cells at risk of energy unbalance. However, there are some mechanisms that could also protect renal cells in the medulla from hypoxic injury. First, increased afferent renal vasoconstriction and GFR reduction with SGLT2 inhibitors limit the substrate load that reaches the distal portions of the nephron. ${ }^{44,87}$ Also, oxygen increments in the proximal renal epithelial cells improve their metabolic activity and ability to reabsorb products more efficiently. Moreover, hypoxia may induce HIF1 and HIF2 signaling pathways in the deep cortex/ outer medulla and enhance the expression of protective genes such as those encoding for heme oxygenase-1. ${ }^{27,87}$ 
Furthermore, activation of HIF2 in fibroblasts could increase the production of erythropoietin, which at the same time increases the hematocrit and renal oxygen delivery. ${ }^{94,100,101}$

\section{Paradigm Shifts in Diabetes Management: Evidence and Limitations in Real World Practice}

The understanding of diabetes mellitus has evolved along with the emerge of SGLT2 inhibitors. There is increase the attention on drugs that can potentially ameliorate cellular and metabolic stress in key tissues which can ultimately serve to decrease the incidence of end-organ damage. It is possible that the modulation of cardiorenal metabolic stress and inflammatory signal pathways play a role. ${ }^{102}$ However, it is challenging to accurately identify the activation of cellular and molecular processes by means of serum or urinary samples. ${ }^{93}$ Further studies are needed to investigate the role of SGLT2 inhibitors in the primary prevention of cardiovascular disease in T2DM. The novel clinical trial DAPA-CKD supports the benefits of SGLT2 inhibitors not only among patients with DM2 but also in patients who do not have this condition. Thus, these medications have major participation in the reduction of progressive kidney disease and associated complications. The Study of the Heart and Kidney Protection with Empagliflozin (EMPA-KIDNEY, NCT03594110) will shed additional light into the role of SGLT2 inhibitors in non-diabetic kidney disease.

Despite the overwhelming body of evidence in favor of SGLT2 inhibitors, major clinical trials have been limited in some aspects. First, they have enrolled predominantly Caucasian participants whereas ethnical minorities such as African American and Latino/Hispanic populations that also suffer from the high burden of T2DM, CKD, and cardiovascular disease have been underrepresented. ${ }^{103}$ Moreover, there is a paucity of patients in the extremes of age ( $>65$ or $<30$ ). The benefit of these medications should be explored in those patients in view of the alarming rates of HTN, obesity, and other risk factors for metabolic and kidney disease among young individuals or the cumulative risk of cardiorenal death and ESKD in patients with advanced age. ${ }^{104-107}$ Second, clinical trials mainly included participants at high cardiovascular risk and the number of adverse events (MACE) may be intrinsically high; overestimating the benefits of SGLT2 inhibitors. Additional studies including patients at low risk for major cardiovascular endpoints would be important to understand the best clinical utility of these medications. Third, despite large randomized clinical trials provide valued insight of the prospective effects of SGLT2 inhibitors, they only offer a short-medium term view, and while the safety profile appears to favor the use of these medications, more data may be needed. ${ }^{108}$ This information could be a help to the incorporation of SGLT2 inhibitors in the practice of general providers and their societal guidelines.

\section{Conclusion}

SGLT2 inhibitors are a novel class of antidiabetic medication that shows great promise in the management of cardiovascular and renal complications in T2DM. Emerging evidence suggests a modulatory role in cellular stress, biochemical balance and inflammation asides from their proven glucose lowering effects. SGLT2 inhibitors have demonstrated to reduce the incidence of atherosclerotic disease including MI, and non-fatal stroke, cardiovascular death and to prevent heart failure related complications in patients with variable degrees of kidney function. Also, SGLT2 inhibitors decrease the progression of DKD, AKI, and kidney replacement therapy needs in large clinical trials. Therefore, SGLT2 inhibitors should be considered in the management of T2DM patients with evidence of cardiovascular or renal disease. Future studies need to focus on the generalizability of the current data by including patients at different stages of cardiac, renal, and metabolic disease.

\section{Author Contributions}

GVR drafted the manuscript. GVR and GNN critically evaluated the literature and content reflected in this manuscript. GNN provided expert consultation for the elaboration of this review. GVR and GNN reviewed and accepted the final version of the manuscript. All authors made substantial contributions to conception and design, acquisition of data, or analysis, and interpretation of data; took part in drafting the article or revising it critically for important intellectual content; agreed to submit to the current journal; gave final approval of the version to be published; and agree to be accountable for all aspects of the work.

\section{Funding}

GNN is supported by a career development award from the NIH (K23DK107908) and is also supported by R01DK127139, R01DK108803, U01HG007278, U01HG00 9610, and 1U01DK116100-01 grants. 


\section{Disclosure}

GNN receives financial compensation as a consultant and is an advisory board member for RenalytixAI and is a cofounder of and owns equity in RenalytixAI. GNN also is an advisory board member for Pensieve Health and is a cofounder of and owns equity in Pensieve Health. GNN has received operational funding from Goldfinch Bio and consulting fees from BioVie Inc, AstraZeneca, Reata and GLG consulting in the past 3 years. The authors report no other conflicts of interest in this work.

\section{References}

1. Saeedi P, Petersohn I, Salpea P, et al. Global and regional diabetes prevalence estimates for 2019 and projections for 2030 and 2045: results from the International Diabetes Federation Diabetes Atlas, 9th edition. Diabetes Res Clin Pract. 2019;157:107843. doi:10.1016/j.diabres.2019.107843

2. Alicic RZ, Rooney MT, Tuttle KR. Diabetic Kidney Disease: challenges, Progress, and Possibilities. Clin J Am Soc Nephrol. 2017;12(12):2032-2045.

3. Thomas B. The Global Burden of Diabetic Kidney Disease: time Trends and Gender Gaps. Curr Diab Rep. 2019;19(4):18.

4. Jiang G, Zhang BB. Glucagon and regulation of glucose metabolism. Am J Physiol Endocrinol Metab. 2003;284(4): E671-8.

5. King P, Peacock I, The DR. UK prospective diabetes study (UKPDS): clinical and therapeutic implications for type 2 diabetes. Br J Clin Pharmacol. 1999;48(5):643-648.

6. Genuth S, Eastman R, Kahn R, et al. Implications of the United kingdom prospective diabetes study. Diabetes Care. 2003;26 (Suppl 1):S28-32.

7. United Kingdom Prospective Diabetes Study (UKPDS). 13: relative efficacy of randomly allocated diet, sulphonylurea, insulin, or metformin in patients with newly diagnosed non-insulin dependent diabetes followed for three years. BMJ. 1995;310 (6972):83-88.

8. Bowker SL, Yasui Y, Veugelers P, Johnson JA. Glucose-lowering agents and cancer mortality rates in type 2 diabetes: assessing effects of time-varying exposure. Diabetologia. 2010;53 (8):1631-1637.

9. Mellbin LG, Malmberg K, Norhammar A, Wedel H, Ryden L, Investigators D. Prognostic implications of glucose-lowering treatment in patients with acute myocardial infarction and diabetes: experiences from an extended follow-up of the Diabetes Mellitus Insulin-Glucose Infusion in Acute Myocardial Infarction (DIGAMI) 2 Study. Diabetologia. 2011;54(6):1308-1317.

10. Antoniades C, Tousoulis D, Marinou K, et al. Effects of insulin dependence on inflammatory process, thrombotic mechanisms and endothelial function, in patients with type 2 diabetes mellitus and coronary atherosclerosis. Clin Cardiol. 2007;30(6):295-300. doi:10.1002/clc.20101

11. Smooke S, Horwich TB, Fonarow GC. Insulin-treated diabetes is associated with a marked increase in mortality in patients with advanced heart failure. Am Heart $J$. 2005;149(1):168-174. doi:10.1016/j.ahj.2004.07.005

12. Margolis DJ, Hoffstad O, Strom BL. Association between serious ischemic cardiac outcomes and medications used to treat diabetes. Pharmacoepidemiol Drug Saf. 2008;17(8):753-759. doi:10.1002/ pds. 1630
13. Kalra S, Shetty KK, Nagarajan VB, Ved JK. Basic and Clinical Pharmaco-Therapeutics of SGLT2 Inhibitors: A Contemporary Update. Diabetes Ther. 2020;11(4):813-833. doi:10.1007/ s13300-020-00789-y

14. Plosker GL. Dapagliflozin: a review of its use in patients with type 2 diabetes. Drugs. 2014;74(18):2191-2209. doi:10.1007/ s40265-014-0324-3

15. Liu B, Wang Y, Zhang Y, Yan B. Mechanisms of Protective Effects of SGLT2 Inhibitors in Cardiovascular Disease and Renal Dysfunction. Curr Top Med Chem. 2019;19 (20): 1818-1849.

16. Nespoux J, Vallon V. Renal effects of SGLT2 inhibitors: an update. Curr Opin Nephrol Hypertens. 2020;29(2):190-198.

17. Ghezzi C, Loo DDF, Wright EM. Physiology of renal glucose handling via SGLT1, SGLT2 and GLUT2. Diabetologia. 2018;61 (10):2087-2097.

18. Poudel RR. Renal glucose handling in diabetes and sodium glucose cotransporter 2 inhibition. Indian $J$ Endocrinol Metab. 2013;17(4):588-593.

19. Vallon V. The proximal tubule in the pathophysiology of the diabetic kidney. Am J Physiol Regul Integr Comp Physiol. 2011;300(5):R1009-22.

20. Vallon V, Gerasimova M, Rose MA, et al. SGLT2 inhibitor empagliflozin reduces renal growth and albuminuria in proportion to hyperglycemia and prevents glomerular hyperfiltration in diabetic Akita mice. Am J Physiol Renal Physiol. 2014;306(2):F194-204.

21. Vallon V. Glucose transporters in the kidney in health and disease. Pflugers Arch. 2020;1:1254.

22. Novikov A, Vallon V. Sodium glucose cotransporter 2 inhibition in the diabetic kidney: an update. Curr Opin Nephrol Hypertens. 2016;25(1):50-58.

23. Ghezzi C, Wright EM. Regulation of the human Na+-dependent glucose cotransporter hSGLT2. Am J Physiol Cell Physiol. 2012;303(3):C348-54

24. Nakamura N, Matsui T, Ishibashi Y, Yamagishi S. Insulin stimulates SGLT2-mediated tubular glucose absorption via oxidative stress generation. Diabetol Metab Syndr. 2015;7:48.

25. Maeda S, Matsui T, Takeuchi M, Yamagishi S. Sodium-glucose cotransporter 2-mediated oxidative stress augments advanced glycation end products-induced tubular cell apoptosis. Diabetes Metab Res Rev. 2013;29(5):406-412.

26. Matsui T, Yamagishi S, Takeuchi M, Ueda S, Fukami K, Okuda S. Irbesartan inhibits advanced glycation end product (AGE)-induced proximal tubular cell injury in vitro by suppressing receptor for AGEs (RAGE) expression. Pharmacol Res. 2010;61(1):34-39.

27. Vallon V, Rose M, Gerasimova M, et al. Knockout of Na-glucose transporter SGLT2 attenuates hyperglycemia and glomerular hyperfiltration but not kidney growth or injury in diabetes mellitus. Am J Physiol Renal Physiol. 2013;304(2):F156-67.

28. Vallon V, Thomson SC. Renal function in diabetic disease models: the tubular system in the pathophysiology of the diabetic kidney. Anпu Rev Physiol. 2012;74:351-375.

29. Poulsen SB, Fenton RA, Rieg T. Sodium-glucose cotransport. Curr Opin Nephrol Hypertens. 2015;24(5):463-469.

30. Maldonado-Cervantes MI, Galicia OG, Moreno-Jaime B, et al. Autocrine modulation of glucose transporter SGLT2 by IL- 6 and TNF-alpha in LLC-PK(1) cells. J Physiol Biochem. 2012;68 (3):411-420.

31. Smith RJ, Goldfine AB, Hiatt WR. Evaluating the Cardiovascular Safety of New Medications for Type 2 Diabetes: time to Reassess? Diabetes Care. 2016;39(5):738-742.

32. Kasichayanula S, Liu X, Lacreta F, Griffen SC, Boulton DW. Clinical pharmacokinetics and pharmacodynamics of dapagliflozin, a selective inhibitor of sodium-glucose co-transporter type 2 . Clin Pharmacokinet. 2014;53(1):17-27. 
33. Dhillon S. Dapagliflozin: A Review in Type 2 Diabetes. Drugs. 2019;79(10):1135-1146.

34. Perkovic V, Jardine MJ, Neal B, et al. Canagliflozin and Renal Outcomes in Type 2 Diabetes and Nephropathy. $N$ Engl J Med. 2019;380(24):2295-2306.

35. Danne T, Biester T, Combined KO. SGLT1 and SGLT2 Inhibitors and Their Role in Diabetes Care. Diabetes Technol Ther. 2018;20 (S2):S269-S77.

36. Lapuerta P, Zambrowicz B, Strumph P, Sands A. Development of sotagliflozin, a dual sodium-dependent glucose transporter $1 / 2$ inhibitor. Diab Vasc Dis Res. 2015;12(2):101-110.

37. Powell DR, Doree D, Jeter-Jones S, Ding ZM, Zambrowicz B, Sands A. Sotagliflozin improves glycemic control in nonobese diabetes-prone mice with type 1 diabetes. Diabetes Metab Syndr Obes. 2015;8:121-127.

38. Zheng SL, Roddick AJ, Aghar-Jaffar R, et al. Association Between Use of Sodium-Glucose Cotransporter 2 Inhibitors, Glucagon-like Peptide 1 Agonists, and Dipeptidyl Peptidase 4 Inhibitors With All-Cause Mortality in Patients With Type 2 Diabetes: A Systematic Review and Meta-analysis. JAMA. 2018;319(15):1580-1591.

39. Zinman B, Wanner C, Lachin JM, et al. Empagliflozin, Cardiovascular Outcomes, and Mortality in Type 2 Diabetes. $N$ Engl J Med. 2015;373(22):2117-2128.

40. Neal B, Perkovic V, Mahaffey KW, et al. Canagliflozin and Cardiovascular and Renal Events in Type 2 Diabetes. $N$ Engl J Med. 2017;377(7):644-657.

41. Wiviott SD, Raz I, Bonaca MP, et al. Dapagliflozin and Cardiovascular Outcomes in Type 2 Diabetes. $N$ Engl $J$ Med. 2019;380(4):347-357.

42. Furtado RHM, Bonaca MP, Raz I, et al. Dapagliflozin and Cardiovascular Outcomes in Patients With Type 2 Diabetes Mellitus and Previous Myocardial Infarction. Circulation. 2019;139(22):2516-2527.

43. Kato ET, Silverman MG, Mosenzon O, et al. Effect of Dapagliflozin on Heart Failure and Mortality in Type 2 Diabetes Mellitus. Circulation. 2019;139(22):2528-2536.

44. Kojima N, Williams JM, Slaughter TN, et al. Renoprotective effects of combined SGLT2 and ACE inhibitor therapy in diabetic Dahl S rats. Physiol Rep. 2015;3:7.

45. Wanner C, Inzucchi SE, Lachin JM, et al. Empagliflozin and Progression of Kidney Disease in Type 2 Diabetes. $N$ Engl $J$ Med. 2016;375(4):323-334.

46. Mahaffey KW, Jardine MJ, Bompoint S, et al. Canagliflozin and Cardiovascular and Renal Outcomes in Type 2 Diabetes Mellitus and Chronic Kidney Disease in Primary and Secondary Cardiovascular Prevention Groups. Circulation. 2019;140 (9):739-750.

47. Seferovic PM, Fragasso G, Petrie M, et al. Sodium-glucose co-transporter 2 inhibitors in heart failure: beyond glycaemic control. The position paper of the Heart Failure Association of the European Society of Cardiology. Eur J Heart Fail. 2020;3.

48. Heerspink HJL, Stefansson BV, Correa-Rotter R, et al. Dapagliflozin in Patients with Chronic Kidney Disease. $N$ Engl $J$ Med. 2020;2:1-58.

49. Sodium-glucose Cotransporter TJ. 2 Inhibitors in Heart Failure: potential Mechanisms of Action, Adverse Effects and Future Developments. Eur Cardiol. 2019;14(1):23-32.

50. Sha S, Polidori D, Heise T, et al. Effect of the sodium glucose co-transporter 2 inhibitor canagliflozin on plasma volume in patients with type 2 diabetes mellitus. Diabetes Obes Metab. 2014;16(11):1087-1095.

51. Lambers Heerspink HJ, de Zeeuw D, Wie L, Leslie B, List J. Dapagliflozin a glucose-regulating drug with diuretic properties in subjects with type 2 diabetes. Diabetes Obes Metab. 2013;15 (9):853-862.
52. Devenny JJ, Godonis HE, Harvey SJ, Rooney S, Cullen MJ, Pelleymounter MA. Weight loss induced by chronic dapagliflozin treatment is attenuated by compensatory hyperphagia in diet-induced obese (DIO) rats. Obesity. 2012;20(8):1645-1652.

53. Cardiovascular SB. Protection by Sodium Glucose Cotransporter 2 Inhibitors: potential Mechanisms. Am J Med. 2017;130(6S):S30-S9.

54. Lei J, Zhao L, Zhang Y, Wu Y, Liu Y. High glucoseinducedpodocyte injury involves activation of mammalian target of rapamycin (mTOR)-induced endoplasmic reticulum (ER) stress. Cell Physiol Biochem. 2018;45(6):2431-2443.

55. Soman S, Raju R, Sandhya VK, et al. A multicellular signal transduction network of AGE/RAGE signaling. J Cell Commun Signal. 2013;7(1):19-23.

56. Eleftheriadis T, Pissas G, Tsogka K, Nikolaou E, Liakopoulos V, Stefanidis I. A unifying model of glucotoxicity in human renal proximal tubular epithelial cells and the effect of the SGLT2 inhibitor dapagliflozin. Int Urol Nephrol. 2020;52(6):1179-1189.

57. Jia G, DeMarco VG, Sowers JR. Insulin resistance and hyperinsulinaemia in diabetic cardiomyopathy. Nat Rev Endocrinol. 2016;12(3):144-153.

58. Jia G, Hill MA, Sowers JR. Diabetic Cardiomyopathy: an Update of Mechanisms Contributing to This Clinical Entity. Circ Res. 2018;122(4):624-638.

59. Aubert G, Martin OJ, Horton JL, et al. The Failing Heart Relies on Ketone Bodies as a Fuel. Circulation. 2016;133(8):698-705.

60. Bedi KC, Snyder NW, Brandimarto J, et al. Evidence for Intramyocardial Disruption of Lipid Metabolism and Increased Myocardial Ketone Utilization in Advanced Human Heart Failure. Circulation. 2016;133(8):706-716.

61. Ferrannini E, Baldi S, Frascerra S, et al. Shift to Fatty Substrate Utilization in Response to Sodium-Glucose Cotransporter 2 Inhibition in Subjects Without Diabetes and Patients With Type 2 Diabetes. Diabetes. 2016;65(5):1190-1195.

62. Ferrannini E, Mark M, Mayoux E. CV Protection in the EMPAREG OUTCOME Trial: A "Thrifty Substrate" Hypothesis. Diabetes Care. 2016;39(7):1108-1114.

63. Garcia-Ropero A, Vargas-Delgado AP, Santos-Gallego CG, Badimon JJ. Inhibition of Sodium Glucose Cotransporters Improves Cardiac Performance. Int J Mol Sci. 2019;20:13.

64. Sakata Y, Masuyama T, Yamamoto K, et al. Calcineurin inhibitor attenuates left ventricular hypertrophy, leading to prevention of heart failure in hypertensive rats. Circulation. 2000;102(18):2269-2275.

65. Vega RB, Bassel-Duby R, Olson EN. Control of cardiac growth and function by calcineurin signaling. J Biol Chem. 2003;278 (39):36981-36984.

66. Baartscheer A, Schumacher CA, Wust RC, et al. Empagliflozin decreases myocardial cytoplasmic $\mathrm{Na}(+)$ through inhibition of the cardiac $\mathrm{Na}(+) / \mathrm{H}(+)$ exchanger in rats and rabbits. Diabetologia. 2017;60(3):568-573.

67. Uthman L, Baartscheer A, Bleijlevens B, et al. Class effects of SGLT2 inhibitors in mouse cardiomyocytes and hearts: inhibition of $\mathrm{Na}(+) / \mathrm{H}(+)$ exchanger, lowering of cytosolic $\mathrm{Na}(+)$ and vasodilation. Diabetologia. 2018;61(3):722-726.

68. Prasad V, Lorenz JN, Miller ML, et al. Loss of NHE1 activity leads to reduced oxidative stress in heart and mitigates high-fat diet-induced myocardial stress. J Mol Cell Cardiol. 2013;65:33-42.

69. Li C, Zhang J, Xue M, et al. SGLT2 inhibition with empagliflozin attenuates myocardial oxidative stress and fibrosis in diabetic mice heart. Cardiovasc Diabetol. 2019;18(1):15.

70. Lee TM, Chang NC, Lin SZ. Dapagliflozin, a selective SGLT2 Inhibitor, attenuated cardiac fibrosis by regulating the macrophage polarization via STAT3 signaling in infarcted rat hearts. Free Radic Biol Med. 2017;104:298-310.

71. Arow M, Waldman M, Yadin D, et al. Sodium-glucose cotransporter 2 inhibitor Dapagliflozin attenuates diabetic cardiomyopathy. Cardiovasc Diabetol. 2020;19(1):7. 
72. Jjv M, Solomon SD, Inzucchi SE, et al. Dapagliflozin in Patients with Heart Failure and Reduced Ejection Fraction. $N$ Engl J Med. 2019;381(21):1995-2008.

73. Ramratnam M, Sharma RK, D’Auria S, et al. Transgenic knockdown of cardiac sodium/glucose cotransporter 1 (SGLT1) attenuates PRKAG2 cardiomyopathy, whereas transgenic overexpression of cardiac SGLT1 causes pathologic hypertrophy and dysfunction in mice. J Am Heart Assoc. 2014;3:4.

74. Kashiwagi Y, Nagoshi T, Yoshino T, et al. Expression of SGLT1 in Human Hearts and Impairment of Cardiac Glucose Uptake by Phlorizin during Ischemia-Reperfusion Injury in Mice. PLoS One. 2015;10(6):e0130605.

75. Li Z, Agrawal V, Ramratnam M, et al. Cardiac sodium-dependent glucose cotransporter 1 is a novel mediator of ischaemia/reperfusion injury. Cardiovasc Res. 2019;115(11):1646-1658.

76. Lim VG, Bell RM, Arjun S, Kolatsi-Joannou M, Long DA, Yellon DM. SGLT2 Inhibitor, Canagliflozin, Attenuates Myocardial Infarction in the Diabetic and Nondiabetic Heart. JACC Basic Transl Sci. 2019;4(1):15-26.

77. Andreadou I, Efentakis P, Balafas E, et al. Empagliflozin Limits Myocardial Infarction in Vivo and Cell Death in Vitro: role of STAT3, Mitochondria, and Redox Aspects. Front Physiol. 2017;8:1077.

78. Hawley SA, Ford RJ, Smith BK, et al. The Na+/Glucose Cotransporter Inhibitor Canagliflozin Activates AMPK by Inhibiting Mitochondrial Function and Increasing Cellular AMP Levels. Diabetes. 2016;65(9):2784-2794.

79. Chang YK, Choi H, Jeong JY, et al. Dapagliflozin, SGLT2 Inhibitor, Attenuates Renal Ischemia-Reperfusion Injury. PLoS One. 2016;11 (7):e0158810

80. Packer M. Activationand inhibition of sodium-hydrogen exchanger is a mechanism that links the pathophysiology and treatment of diabetes mellitus with that of heart failure. Circulation. 2017;136(16):1548-1559.

81. Ruggenenti P, Porrini EL, Gaspari F, et al. Glomerular hyperfiltration and renal disease progression in type 2 diabetes. Diabetes Care. 2012;35(10):2061-2068.

82. Baba M, Shimbo T, Horio M, et al. Longitudinal Study of the Decline in Renal Function in Healthy Subjects. PLoS One. 2015;10(6):e0129036.

83. Rowe JW, Andres R, Tobin JD, Norris AH, Shock NW. The effect of age on creatinine clearance in men: a cross-sectional and longitudinal study. J Gerontol. 1976;31(2):155-163.

84. Chagnac A, Zingerman B, Rozen-Zvi B, Herman-Edelstein M. Consequences of Glomerular Hyperfiltration: the Role of Physical Forces in the Pathogenesis of Chronic Kidney Disease in Diabetes and Obesity. Nephron. 2019;143(1):38-42.

85. Velagapudi C, Bhandari BS, Abboud-Werner S, Simone S, Abboud HE, Habib SL. The tuberin/mTOR pathway promotes apoptosis of tubular epithelial cells in diabetes. J Am Soc Nephrol. 2011;22(2):262-273.

86. Shankland SJ, Scholey JW, Ly H, Thai K. Expression of transforming growth factor-beta 1 during diabetic renal hypertrophy. Kidney Int. 1994;46(2):430-442.

87. Nespoux J, Vallon V. SGLT2 inhibition and kidney protection. Clin Sci (Lond). 2018;132(12):1329-1339.

88. Fattah H, Layton A, How VV. Do Kidneys Adapt to a Deficit or Loss in Nephron Number? Physiology. 2019;34(3):189-197.

89. Yiu WH, Lin M, Tang SC. Toll-like receptor activation: from renal inflammation to fibrosis. Kidney Int Suppl. 2014;4(1):20-25.

90. Ashrafi Jigheh Z, Ghorbani Haghjo A, Argani $H$, et al. Empagliflozin alleviates renal inflammation and oxidative stress in streptozotocin-induced diabetic rats partly by repressing HMGB1-TLR4 receptor axis. Iran J Basic Med Sci. 2019;22 (4):384-390.
91. Gallo LA, Ward MS, Fotheringham AK, et al. Once daily administration of the SGLT2 inhibitor, empagliflozin, attenuates markers of renal fibrosis without improving albuminuria in diabetic db/db mice. Sci Rep. 2016;6:26428.

92. Petrykiv SI, Laverman GD, de Zeeuw D, Heerspink HJL. The albuminuria-lowering response to dapagliflozin is variable and reproducible among individual patients. Diabetes Obes Metab. 2017;19(10):1363-1370.

93. Dekkers CCJ, Petrykiv S, Laverman GD, Cherney DZ, Gansevoort RT, Heerspink HJL. Effects of the SGLT-2 inhibitor dapagliflozin on glomerular and tubular injury markers. Diabetes Obes Metab. 2018;20(8):1988-1993.

94. Fu Q, Colgan SP, Shelley CS. Hypoxia: the Force that Drives Chronic Kidney Disease. Clin Med Res. 2016;14(1):15-39.

95. Takaori K, Nakamura J, Yamamoto S, et al. Severity and Frequency of Proximal Tubule Injury Determines Renal Prognosis. J Am Soc Nephrol. 2016;27(8):2393-2406.

96. Fujita Y, Doi Y, Hamano T, et al. Low erythropoietin levels predict faster renal function decline in diabetic patients with anemia: a prospective cohort study. Sci Rep. 2019;9(1):14871.

97. Babazono T, Hanai K, Suzuki K, et al. Lower haemoglobin level and subsequent decline in kidney function in type 2 diabetic adults without clinical albuminuria. Diabetologia. 2006;49 (6):1387-1393.

98. Sano M, Goto S. Possible Mechanism of Hematocrit Elevation by Sodium Glucose Cotransporter 2 Inhibitors and Associated Beneficial Renal and Cardiovascular Effects. Circulation. 2019;139(17):1985-1987.

99. Asada N, Takase M, Nakamura J, et al. Dysfunction of fibroblasts of extrarenal origin underlies renal fibrosis and renal anemia in mice. J Clin Invest. 2011;121(10):3981-3990.

100. Yang X, Chen B, Liu T, Chen X. Reversal of myofibroblast differentiation: a review. Eur J Pharmacol. 2014;734:83-90. doi:10.1016/j.ejphar.2014.04.007

101. Meran S, Steadman R. Fibroblasts and myofibroblasts in renal fibrosis. Int J Exp Pathol. 2011;92(3):158-167. doi:10.1111/ j.1365-2613.2011.00764.x

102. Yaribeygi H, Butler AE, Atkin SL, Katsiki N, Sahebkar A. Sodium-glucose cotransporter 2 inhibitors and inflammation in chronic kidney disease: possible molecular pathways. J Cell Physiol. 2019;234(1):223-230. doi:10.1002/jcp.26851

103. Neumiller JJ, Kalyani RR. How Does CREDENCE Inform Best Use of SGLT2 Inhibitors in CKD? Clin J Am Soc Nephrol. 2019;14(11):1667-1669. doi:10.2215/CJN.05340419

104. Stevens LA, Viswanathan G, Weiner DE. Chronic kidney disease and end-stage renal disease in the elderly population: current prevalence, future projections, and clinical significance. Adv Chronic Kidney Dis. 2010;17(4):293-301. doi:10.1053/j.ackd.2010.03.010

105. Kurella Tamura M. Incidence, management, and outcomes of end-stage renal disease in the elderly. Curr Opin Nephrol Hypertens. 2009;18(3):252-257. doi:10.1097/MNH.0b013e328326f3ac

106. Murthy VL, Abbasi SA, Siddique J, et al. Transitions in Metabolic Risk and Long-Term Cardiovascular Health: coronary Artery Risk Development in Young Adults (CARDIA) Study. $J$ Am Heart Assoc. 2016;5(10):10. doi:10.1161/JAHA.116.003934

107. Cheung EL, Bell CS, Samuel JP, Poffenbarger T, Redwine KM, Samuels JA. Race and Obesity in Adolescent Hypertension. Pediatrics. 2017;139(5):5. doi:10.1542/peds.2016-1433

108. Zhang X-L, Zhu -Q-Q, Chen Y-H, et al. Cardiovascular Safety, Long-Term Noncardiovascular Safety, and Efficacy of SodiumGlucose Cotransporter 2 Inhibitors in Patients With Type 2 Diabetes Mellitus: A Systemic Review and Meta-Analysis With Trial Sequential Analysis. J Am Heart Assoc. 2018;7(2):2. doi:10.1161/JAHA.117.007165 


\section{Publish your work in this journal}

The International Journal of Nephrology and Renovascular Disease is an international, peer-reviewed open-access journal focusing on the pathophysiology of the kidney and vascular supply. Epidemiology, screening, diagnosis, and treatment interventions are covered as well as basic science, biochemical and immunological studies. The manuscript management system is completely online and includes a very quick and fair peer-review system, which is all easy to use. Visit http://www.dovepress.com/testimonials.php to read real quotes from published authors.

Submit your manuscript here: https://www.dovepress.com/international-journal-of-nephrology-and-renovascular-disease-journal 